\title{
Effect of nodularity on mechanical properties and fracture of ferritic spheroidal graphite iron
}

\author{
*Ze-hua Wang ${ }^{1}$, Xin Zhang ${ }^{1}$, Fei-long Xu ${ }^{1}$, Kun-cai Qian ${ }^{2}$, Kai-min Chen ${ }^{2}$ \\ 1. College of Mechanics and Materials, Hohai University, Nanjing 211100, Jiangsu, China \\ 2. CRRC Qishuyan Institute Co., Ltd., Changzhou, 213011, Jiangsu, China
}

\begin{abstract}
Ferritic spheroidal graphite irons with nodularity from $72 \%$ to $96 \%$ were prepared. The relationship between the nodularity and the mechanical properties of the ferritic spheroidal graphite iron was investigated. The effect of nodularity on the mechanical properties and tensile fracture of the cast iron were studied. Results showed that the tensile strength $R_{\mathrm{m}}$, yield strength $R_{\mathrm{p} 0.2}$, elongation to failure $A_{5}$, and impact energy $K V_{2}$ of the cast iron had a good linear relationship with its nodularity. Nodularity and annealing treatment would obviously affect the fracture characteristics of ferritic spheroidal graphite iron. The annealed ferritic spheroidal graphite iron with $93 \%$ nodularity showed a completely ductile rupture. With the decrease of nodularity from $93 \%$ to $72 \%$, the cleavage fracture area ratio increased gradually from $0 \%$ to $8.3 \%$. Compared with as-cast ferritic spheroidal graphite iron, annealing treatment reduced the cleavage fracture area of the ferritic spheroidal graphite iron.
\end{abstract}

Key words: ductile iron; nodularity; mechanical property; fracture

CLC numbers: TG143.5 Document code:A Article ID: 1672-6421(2019)06-386-07

\begin{abstract}
A $\mathrm{s}$ an important engineering material, spheroidal graphite cast iron has been widely applied in industry, with a global production in 2017 of more than 2,643 million tons ${ }^{[1]}$. The spheroidal graphite particles in the spheroidal graphite cast iron are characteristics of its microstructure. Nodularity, as a parameter representing the shape of the graphite particles in group, is one of the most important quality indications of spheroidal graphite cast irons ${ }^{[2]}$. The influence of nodularity on the tensile property, impact property and fatigue strength of spheroidal graphite cast irons have been extensively studied ${ }^{[3-6]}$. The results indicated that the shape of graphite particles has obvious effect on the mechanical properties of spheroidal graphite cast irons ${ }^{[7-8]}$. Generally, with the decrease of nodularity, the mechanical properties decline ${ }^{[9-13]}$. However, the exact relations between the graphite particles's shape and mechanical properties are not declared up to now. The main reason is that the mechanical properties of spheroidal graphite cast irons are not only related to the shape of graphite particles, but also to its matrix, including its microstructure and the chemical composition. The reported research studies
\end{abstract}

\section{* Ze-hua Wang}

Male, Professor, mainly engages in research of casting alloys and casting processes, has publised more than 160 technological papers and developed an international standard ISO $945-4$ as the project leader.

E-mail: zhwang@hhu.edu.cn

Received: 2019-06-24; Accepted: 2019-08-30 were based on samples taken from different melts or different locations within a casting with different thicknesses and different solidification rates ${ }^{[14-15]}$. In these cases, it is too difficult to tell the effect of the graphite particles or of the matrix.

In order to control the influence of nodularity and the matrix on mechanical properties of spheroidal graphite cast iron, samples having different nodularity were prepared. The effect of nodularity on the mechanical properties was investigated and the tensile fracture mechanism of a ferritic spheroidal graphite cast iron was analyzed.

\section{Experimental}

\subsection{Material preparation}

The chemical composition of a spheroidal graphite cast iron is shown in Table 1. Q10 pig iron, steel scrap and foundry returns, as raw materials, were melted in a medium-frequency induction furnace. The sandwich method of spheroidizing process was employed to treat the liquid metal. Samples with different nodularity were obtained by regulating the addition amount of nodulizer during the spheroidizing treatment process. The nodulizer contains 5.5wt.\%-6.6wt.\% $\mathrm{Mg}$ and less than $1.0 \%$ RE. The particle sizes were in the range of 5-15 $\mathrm{mm}$. Silicon-barium inoculants and post-inoculation were used in this work. The pouring temperature was controlled in the range of $1,400-1,360{ }^{\circ} \mathrm{C}$. Type-Y 
Table 1: Chemical composition of the test pieces (wt.\%)

$\begin{array}{ccccccccc}\mathbf{C} & \text { Si } & \text { Mn } & \mathbf{P} & \mathbf{S} & \mathbf{R e} & \mathrm{Mg} & \mathrm{Fe} \\ 3.65 & 2.56 & 0.18 & 0.03 & <0.02 & \leq 0.01 & 0.021-0.036 & \text { Bal. }\end{array}$

blocks were cast in sand molds and the test pieces were cut from the bottom of the block, as shown in Fig. 1.

In order to get a uniform ferritic matrix, an annealing process, as shown in Fig. 2, was applied to the samples to eliminate the pearlite and cementite. The annealing treatment was carried out in an electrical furnace.

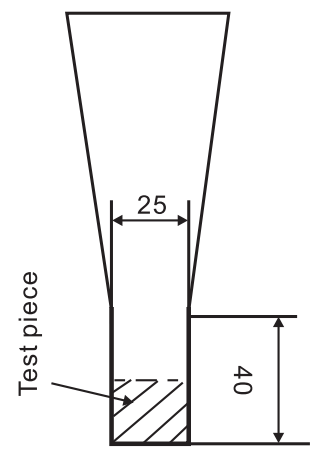

Fig. 1: Type-Y block and location of test piece

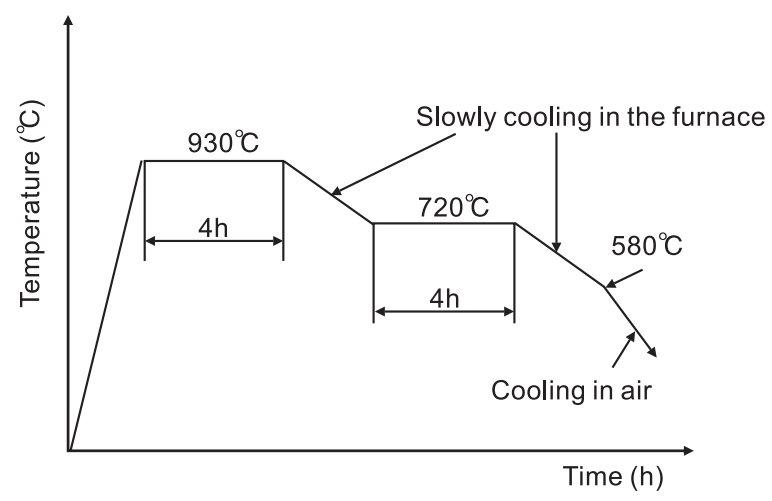

Fig. 2: Annealing process for test samples

\subsection{Microstructure and mechanical property analysis}

1.2.1 Nodularity evaluation

The microstructure and the graphite shape were observed under a LEICA DM4000 M optical microscopy. The nodularity was evaluated using the LAMOS master V18.3 software and the average value was obtained from 10 fields of views randomly selected in each sample.

\subsubsection{Mechanical property test}

(1) Tensile property test

The tensile property test was performed according to GB/ T228-2002 at a strain rate of $0.25 \times 10^{-3}$ using a CMT5205 tensile testing machine. The diameter of the sample was 10 $\mathrm{mm}$ and the gauge length was $50 \mathrm{~mm}$. The surface roughness of the sample was $0.8-1.6 \mu \mathrm{m}$.
(2) Impact test

The size of the V-notched sample was $10 \mathrm{~mm} \times 10 \mathrm{~mm} \times 55$ $\mathrm{mm}$. The $\mathrm{V}$-notch of all samples was checked using a profile projector. Impact energy was the average value of three samples.

\subsubsection{Fracture morphology analysis}

The tensile fracture morphology was analyzed under a HITACHI S-3600 scanning electron microscope. Image analysis software Pro Plus was used to measure the area of the cleavage plane in the fracture. The average area ratio of the cleavage plane was obtained from 10 fields of views in a sample. The field was $420 \mu \mathrm{m} \times 300 \mu \mathrm{m}$, which is selected at random on the tensile fracture of the sample.

\section{Results and discussion}

\subsection{Effect of nodularity on mechanical properties}

Figure 3 shows the relationship between the mechanical properties and nodularity of a ferritic spheroidal graphite iron. Figure 3 demonstrates that with the increase of nodularity, the tensile strength, yield strength, elongation to failure and impacting energy generally increased. However, the properties of the samples having the same nodularity fluctuated a lot. For example, among 6 samples having nodularity of $92 \%$, the tensile strength changed in the range from $415 \mathrm{MPa}$ to $424 \mathrm{MPa}$, and the elongation to failure from $18 \%$ to $20 \%$.

The fitted equations of nodularity $p_{\text {nod }}$ vs tensile strength $R_{\mathrm{m}}$, yield strength $R_{\mathrm{p} 0.2}$, elongation to failure $A_{5}$, and impact energy $K V_{2}$, were as follows:

$$
\begin{array}{ll}
R_{\mathrm{m}}=99.1 p_{\text {nod }}+326.7 & R^{2}=0.77 \\
R_{\mathrm{p} 0.2}=35.9 p_{\text {nod }}+247.0 & R^{2}=0.32 \\
A_{5}=19.6 p_{\text {nod }}+0.86 & R^{2}=0.50 \\
K V_{2}=9.49 p_{\text {nod }}+6.44 & R^{2}=0.54
\end{array}
$$

The correlation coefficients $R^{2}$ of the fitted equations were 0.77 , $0.32,0.50$ and 0.54 , respectively. It seems that the mechanical properties of a ferritic spheroidal graphite iron had a low correlation with nodularity. As is known, the measured value of the mechanical properties of a ferritic spheroidal graphite iron is not only affected by nodularity, but also by many other factors, such as, chemical composition, matrix, machining quality of the sample, manual operation, etc. In this work, in order to reduce the distraction from other factors, the raw materials, liquid metal, pouring process, location of the test pieces, surface roughness of the test samples and the notch shape of impact sample were kept as similar as possible. A precise annealing treatment was applied to the samples and all of samples were heat-treated together to obtain an identical matrix structure. However, there are always some unavoidable factors which would result in the disturbance of the mechanical property.

These random interference factors can obviously affect the test results of a single sample. According to statistical theory, increasing the number of samples would reduce the effects of interference. The greater the number of samples, the more stable the obtained statistical data would be. An average tensile 

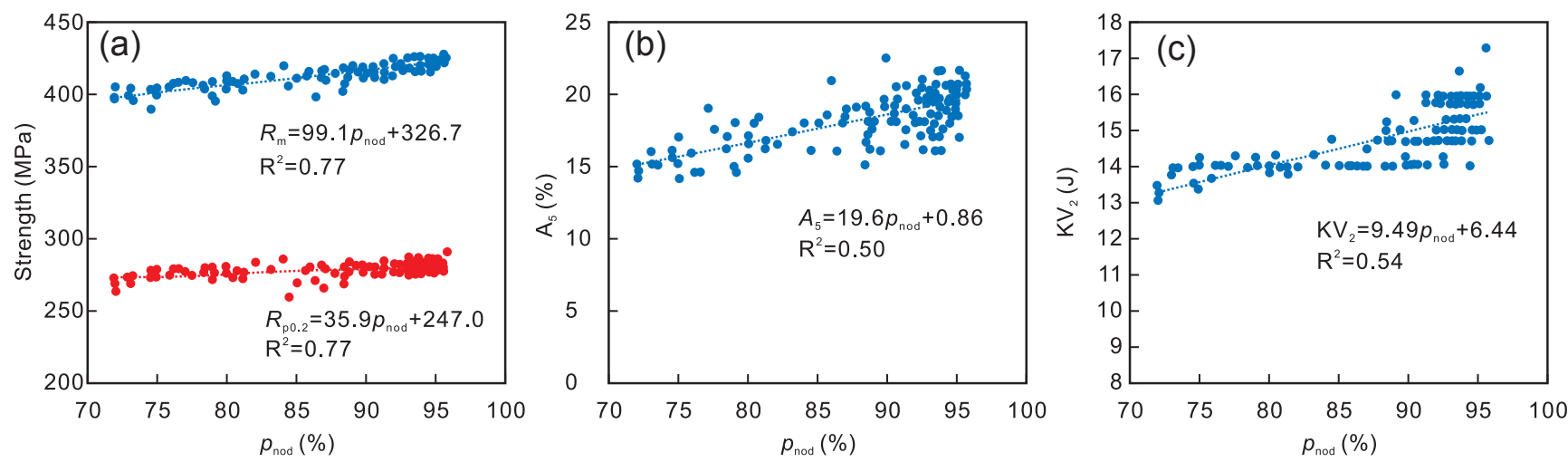

Fig. 3: Relationship between mechanical properties and nodularity of ferritic spheroidal graphite iron:

(a) tensile strength; (b) elongation; (c) impact energy

strength $R_{\mathrm{m}}$, average yield strength $R_{\mathrm{p} 0.2}$, elongation to failure $A_{5}$ and average impact energy $K V_{2}$ were obtained from the test results of Fig. 3 for the samples which had the same nodularity and the number of the samples was equal to and more than 3. The relationships between nodularity and the average mechanical properties were as shown in Fig. 4. The fitting equation of nodularity $p_{\text {nod }}$ and $R_{\mathrm{m}}, R_{\mathrm{p} 0.2}, A_{5}$, and $K V_{2}$ could be expressed as follows:

$$
\begin{array}{ll}
R_{\mathrm{m}}=104.1 p_{\text {nod }}+321.9 & \mathrm{R}^{2}=0.95 \\
R_{\mathrm{p} 0.2}=42.3 p_{\text {nod }}+241.3 & \mathrm{R}^{2}=0.84 \\
A_{5}=20.5 p_{\text {nod }}+0.17 & \mathrm{R}^{2}=0.89 \\
K V_{2}=8.75 p_{\text {nod }}+7.04 & \mathrm{R}^{2}=0.80
\end{array}
$$

Obviously, the fitting equation of nodularity $p_{\text {nod }}$ and average mechanical properties increased the correlation coefficient $\mathrm{R}^{2}$. The correlation coefficient $\mathrm{R}^{2}$ of the fitting equation of nodularity $p_{\text {nod }}$ and average tensile strength increased from 0.77 to 0.95 , average yield strength increased from 0.32 to 0.84 , average elongation to failure increased from 0.50 to 0.89 and average impact energy increased from 0.54 to 0.80 . Therefore, it could be concluded that the tensile strength, yield strength, elongation to failure and impact energy of ferritic spheroidal graphite iron had a good linear relationship with its nodularity, when the nodularity is in the range of $72 \%$ to $96 \%$. With the decrease of the nodularity, the tensile strength, yield strength, elongation to failure and impact energy of ferritic spheroidal graphite cast iron gradually decreased. It is easy to understand that the lower nodularity means the more irregular graphite particles, which will result in stress concentration, destroying the continuity of the matrix and decrease the mechanical properties of the metal.

From Fig. 3(a) and Fig. 4(a), it can be found that with the decrease of the nodularity, the decline of the tensile strength of ferritic spheroidal graphite iron was quicker than its yield strength. The decline rate of the average tensile strength was 2.46 times that of its average yield strength. As is known, ferrite has very good ductility compared to pearlite. Stress concentration caused by the existence of small graphite particles, especially irregular graphite particles, would promote micro-zone plastic deformation. Its notch effect increased the strength of ferritic matrix locally and offset the negative effect of irregular graphite particles on yield strength to some extent.

The graphite particles in spheroidal graphite iron can be considered as the cavity or inclusions in the matrix. This is because the strength of the graphite is far lower than that of the matrix and the bonding strength between graphite particles and the matrix is poor, the graphite particles can easily break away from the matrix. If the graphite particles in spheroidal graphite iron were treated as cavities or inclusions in the metal, it can be supposed that total mechanical load was on the spheroidal
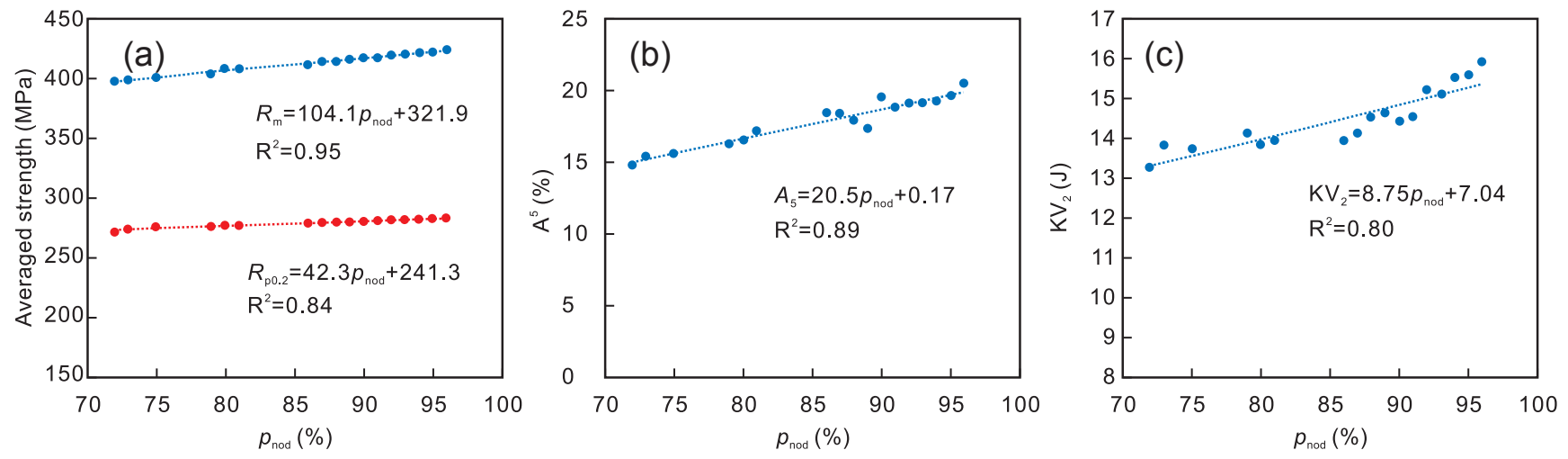

Fig. 4: Relationship between the average value of (a) $\overline{R_{\mathrm{m}}}, \overline{R_{\mathrm{p} 0.2}}$, (b) $\overline{A_{5}}$, (c) $\overline{K V_{2}}$ and nodularity of ferritic spheroidal graphite iron 
graphite iron. The effect of graphite particles on the mechanical property of the ferritic spheroidal graphite iron can be treated as the effect of a cavity or inclusion in the property of the metals.

Cavities in metals cause stress concentrations in the principal stress direction ${ }^{[16-18]}$. As shown in Fig. 5, the stress concentration factor is closely related to the shape of the cavity. An elliptical hole would produce a greater stress concentration factor than a round hole. The bigger the $\mathrm{a} / \mathrm{b}$ value is, the greater the stress concentration factor $k_{0}$ is ${ }^{[19]}$. So, graphite particles in spheroidal graphite iron would cause stress concentration and decrease the strength of the metals. The lower nodularity means more irregular graphite particles and/or worse roundness of the graphite particles in spheroidal graphite iron, which would result in more high-stress areas and/or increase the stress in the matrix around the graphite

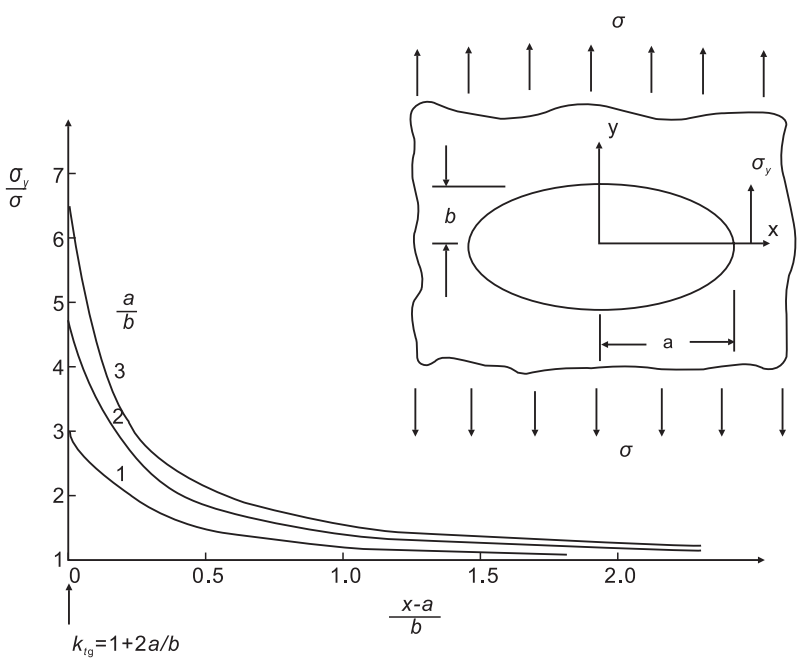

Fig. 5: $\sigma_{\mathrm{y}}$ stress with distance $(\mathrm{x}-\mathrm{a})$ away from an elliptical hole in uniaxial tension ${ }^{[18]}$
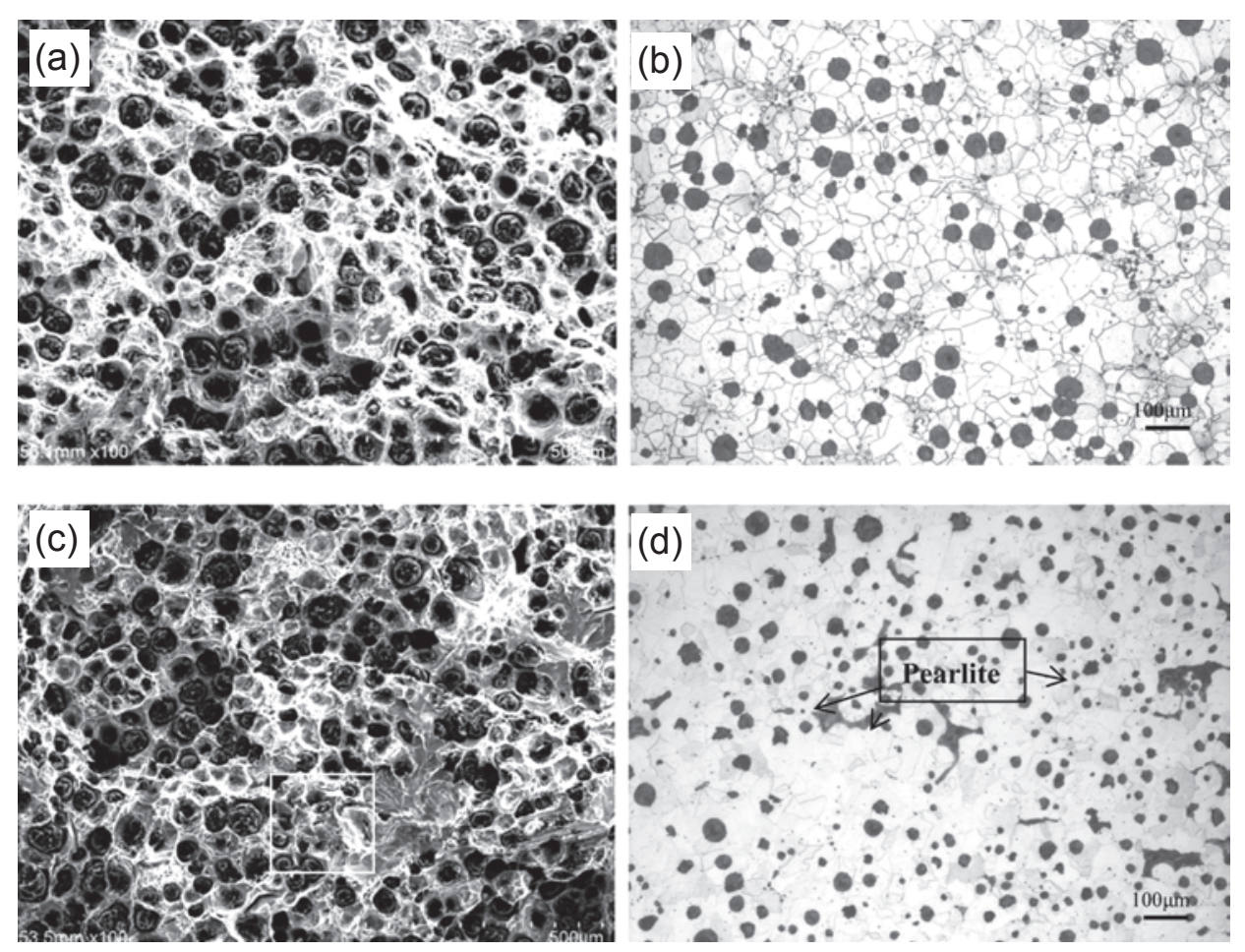

Fig. 6: Fractures and microstructures of ferritic spheroidal graphite cast iron (93\% nodularity): (a) Fracture of annealed
sample; (b) Microstructure of annealed sample; (c) Fracture of as-cast sample; (d) Microstructure of as-cast sample

Fig. 6: Fractures and microstructures of ferritic spheroidal graphite cast iron (93\% nodularity): (a) Fracture of annealed
sample; (b) Microstructure of annealed sample; (c) Fracture of as-cast sample; (d) Microstructure of as-cast sample particles. This will reduce the strength of the metal.

Research has discovered that the stress concentration was not only related to the shape of the hole or inclusion particle, but also closely related to its distribution ${ }^{[20]}$. When the ratio of the distance between two particles $l$ and the radius of the particles $a$ is close to 2 , the stress concentration obviously increases ${ }^{[20]}$. When the $l /$ $a$ value is close to 6 , the stress concentration is equal to the stress concentration caused by a single particle ${ }^{[17-18]}$. This means that the greater the distance between graphite particles, the less the stress concentration in the matrix. High stress concentration would change the stress distribution. The tensile stress would be changed into a complex stress, especially when the area is near irregular graphite particles, which would increase the brittleness and reduce the plasticity of the metal. For spheroidal graphite iron, the carbon content is usually controlled near the eutectic composition, the volume ratio of the graphite particles is about $10 \%-12 \%$. As the content of graphite is nearly fixed, it can be concluded that the more uniform the distribution of graphite particles, the greater the distance between the graphite particles, therefore the lower the stress concentration would be within the material. As a result, the uniform distribution of graphite particles is beneficial to the mechanical properties of spheroidal graphite irons.

\subsection{Effect of nodularity on fracture mechanism}

Figure 6 shows the tensile fractures and microstructure of ascast and annealed ferritic spheroidal graphite iron with 93\% nodularity. The annealed iron showed a typical ductile fracture, full of dimples around the graphite particles, as shown in Fig. 6(a). Figure 6(b) shows that the annealed iron was $100 \%$ ferritic matrix. Some cleavage planes with large numbers of dimples could be found in the tensile fracture of the as-cast 
iron, as seen in Fig. 6(c). Small amounts of pearlite can be found in the as-cast iron as shown in Fig. 6(d). This is due to the existence of pearlite in the matrix. Pearlite has a lower plasticity than ferrite, and promotes the formation of cleavage fractures. Annealing treatment not only eliminated the pearlite in the matrix, but also could remove supersaturated carbon atoms from ferrite, reducing its lattice distortion and increasing the plasticity of the ferrite. So, high temperature annealing treatment would change the tensile fracture mechanism of ferritic spheroidal graphite iron.

Figure 7 shows the tensile fractures of annealed ferritic spheroidal graphite iron with different nodularity from $91 \%$ to $75 \%$. The images on the right show the locally enlarged images taken from the images on the left. In Fig. 7 (a), the sample had
91\% nodularity. A large number of dimples and some cleavage plane areas could be found in the fracture. With the decrease of nodularity from $91 \%$ to $75 \%$, the cleavage fracture area increased gradually, as shown in Fig. 7 (a) to (d). Many cleavage fracture areas appeared in the fracture of annealed ferritic spheroidal graphite iron with $75 \%$ nodularity, as shown in Fig. 7 (d).

Figure 8 shows the cleavage fracture area ratio of annealed ferritic spheroidal graphite iron having different levels of nodularity. No cleavage fracture was found in annealed ferritic spheroidal graphite iron with $93 \%$ nodularity, as stated before. The cleavage fracture area ratios of annealed ferritic spheroidal graphite iron with $91 \%, 89 \%, 87 \%, 85 \%, 79 \%$ and $75 \%$ nodularity were $3.0 \%, 3.5 \%, 4.5 \%, 4.6 \%, 6.3 \%$ and $8.3 \%$, respectively.
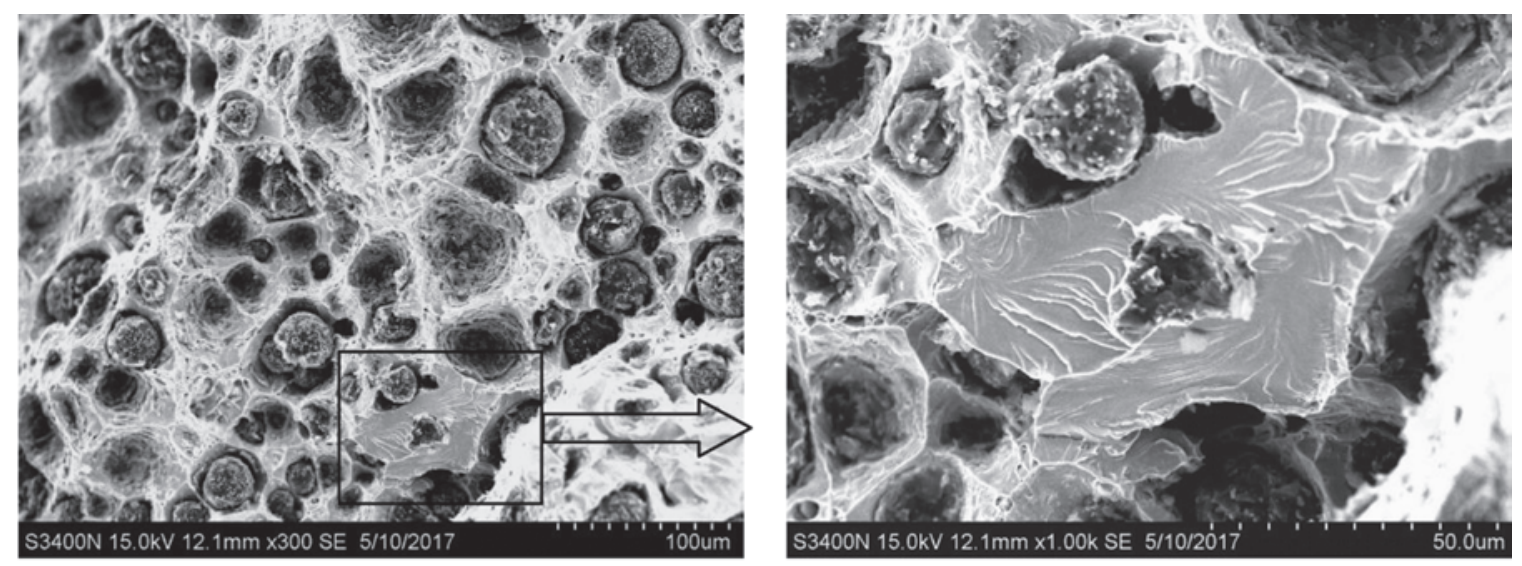

(a) $91 \%$ nodularity
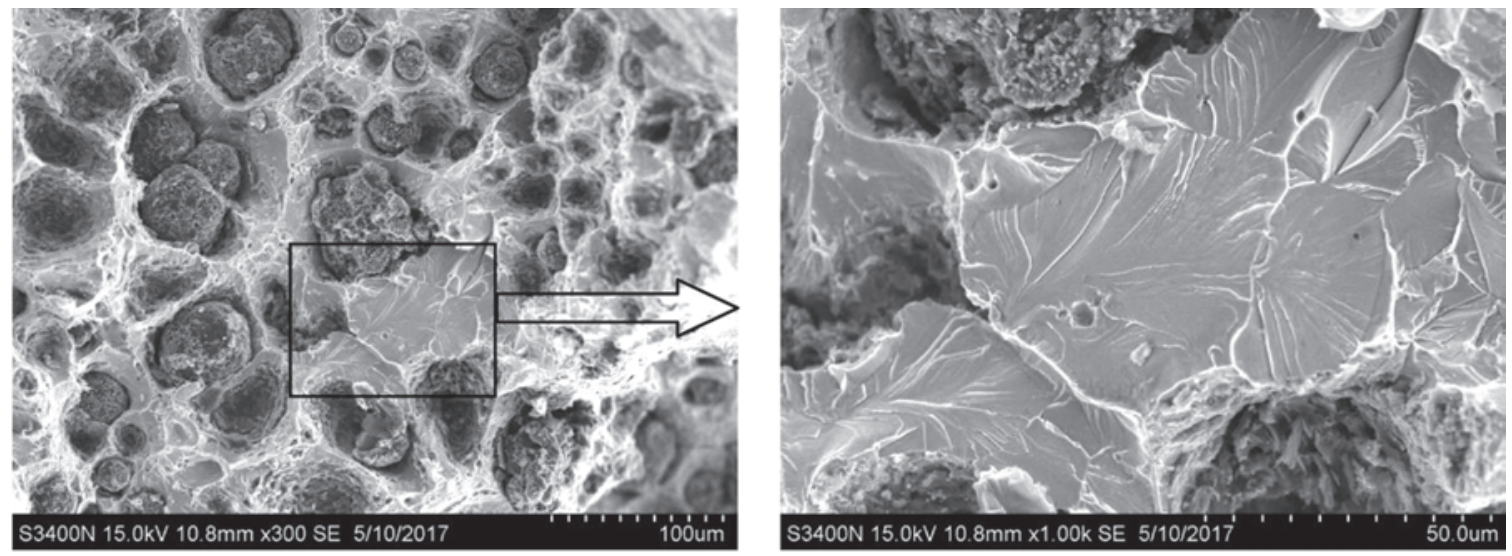

(b) $87 \%$ nodularity
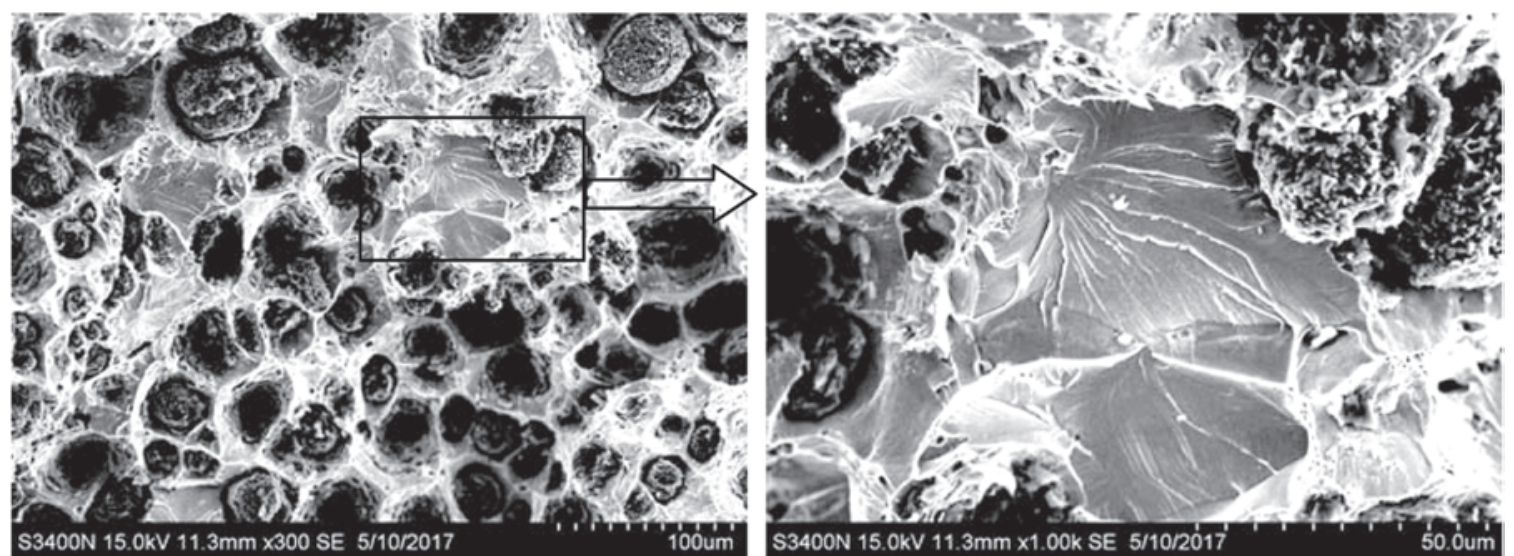

(c) $79 \%$ nodularity 

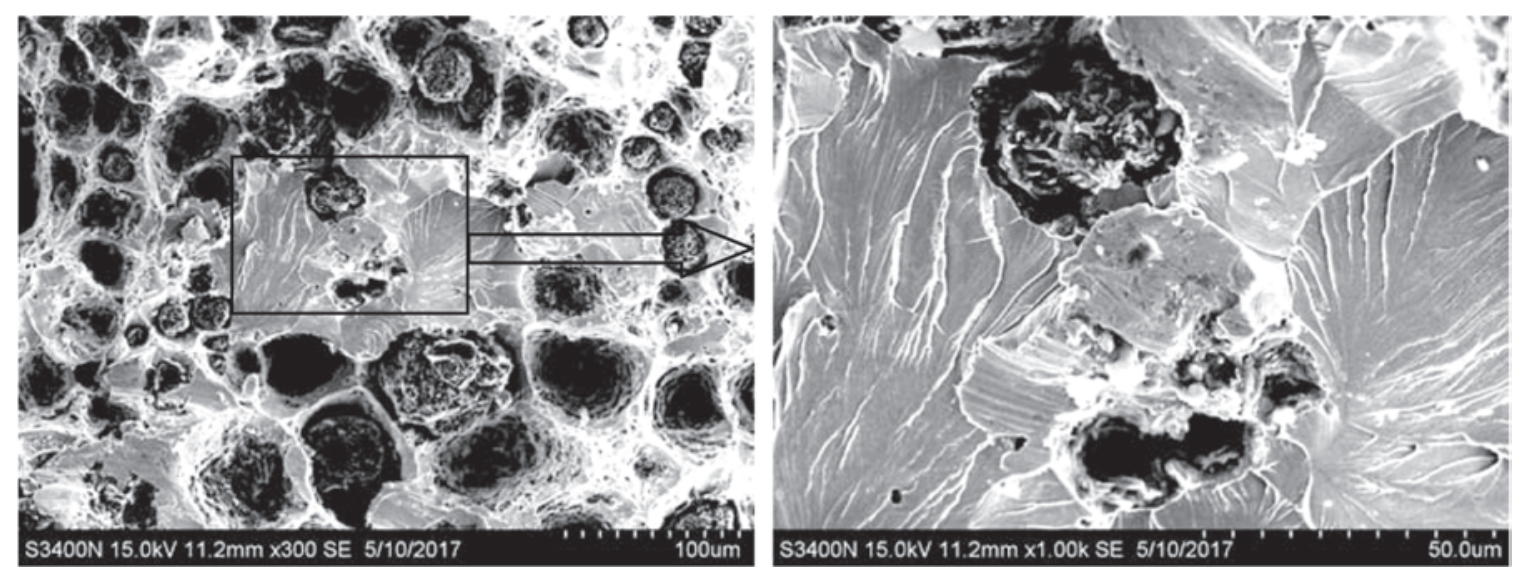

(d) $75 \%$ nodularity

Fig. 7: Tensile fractures of annealed ferritic spheroidal graphite iron: (a) $91 \%$ nodularity; (b) $87 \%$ nodularity; (c) $79 \%$ nodularity; (d) $75 \%$ nodularity

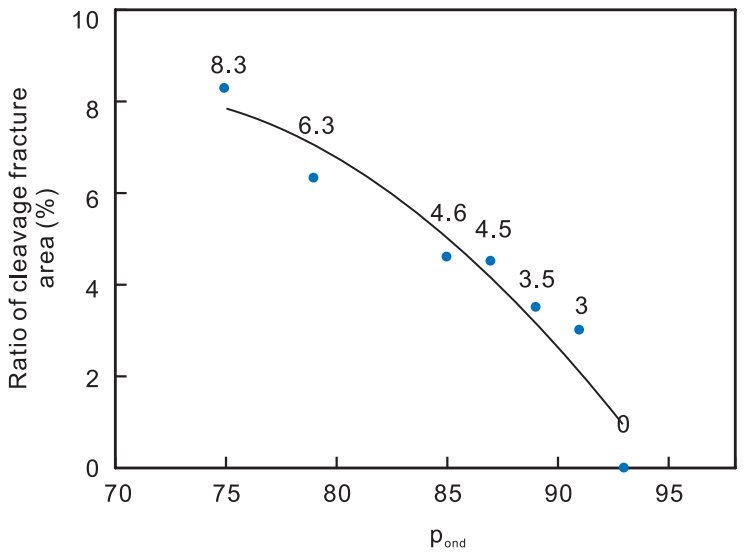

Fig. 8: Cleavage fracture area ratio and nodularity of annealed ferritic spheroidal graphite iron

With the decrease of nodularity, the cleavage fracture area ratio increased gradually. This was caused by the notch effects of irregular graphite particles ${ }^{[21]}$. The irregular angle of graphite particles could change the stress distribution of the matrix from a single tensile stress into a two- or threedimensional stress, which makes the crystal-plane slipping of ferrite difficult. In turn, more cleavage fracture would appear. As is known, the lower the nodularity, the more the irregular angles exist, the greater the notch effect. So it can be concluded that the lower the nodularity, the more the cleavage fracture area appears, and the worse the toughness.

\section{Conclusions}

The effect of nodularity on the mechanical properties and tensile fracture of ferritic spheroidal graphite iron was studied. The following conclusions can be obtained:

(1) The tensile strength $R_{\mathrm{m}}$, yield strength $R_{\mathrm{p} 0.2}$, elongation to failure $A_{5}$ and impact energy $K V_{2}$ of ferritic spheroidal graphite iron all had a good linear relationship with nodularity $p_{\text {nod }}$. The fitting equations of the average $R_{\mathrm{m}}, R_{\mathrm{p} 0.2}, A_{5}$ and $K V_{2}$ were $R_{\mathrm{m}}$ $=104.1 p_{\text {nod }}+321.9, R_{\mathrm{p} 0.2}=42.3 p_{\text {nod }}+241.3, A_{5}=20.5 p_{\text {nod }}+0.17$ and $K V_{2}=8.75 p_{\text {nod }}+7.04$, respectively, and their correlation coefficients $\mathrm{R}^{2}$ were $0.95,0.84,0.89$ and 0.80 , respectively.

(2) Nodularity would clearly affect the fracture characteristics of annealed ferritic spheroidal graphite iron. With decrease of nodularity from $93 \%$ to $72 \%$, the cleavage fracture area ratio increased gradually from $0 \%$ to $8.3 \%$.

(3) Annealing treatment could reduce the cleavage fracture of ferritic spheroidal graphite iron. Annealed ferritic spheroidal graphite iron with $93 \%$ nodularity shows a wholly ductile rupture.

\section{References}

[1] A modern casting staff report, Worldwide casting production grew by $5.3 \%$ during a busy 2017. Modern Casting, 2018, 12: 23-26.

[2] ISO 1083:2018, Spheroidal graphite cast irons-Classification. Switzerland: ISO copyright office, 2018.

[3] Al-Ghonamy A I, Ramadan M, Fathy N, et al. Effect of graphite nodularity on mechanical properties of ductile iron for waterworks fittings and accessories. International Journal of Civil \& Environmental Engineering, 2010, 10 (3):1-5.

[4] Mourujarvi A, Widell K, Saukkonen T, et al. Influence of chunky graphite on mechanical and fatigue properties of heavysection cast iron. Fatigue \& Fracture of Engineering Materials \& Structures, 2010, 32 (5): 379-390.

[5] Cocco V D, lacoviello F. Pearlitic ductile cast irons: Fatigue initiation micromechanisms. Procedia Engineering, 2015, 109: 465-472.

[6] Yu G L, Zhang W P, Han W Y. Micromechanic fatigue crack propagation of ductile iron. Chinese Journal of Mechanical Engineering, 1994, 30(5): 35-39 (In Chinese).

[7] Yung N P, Chen C F, Chih H C. Effects of graphite shape on thermal fatigue property of thin wall graphitic cast irons. Key Engineering Materials, 2011, 457: 398-403.

[8] Liu Z, Chen W P and Deng Y. Influence of cooling rate and antimony addition content on graphite morphology and mechanical properties of a ductile iron. China Foundry, 2012,9 (2): 114-118.

[9] lacoviello F, Cocco V D, Cavallini M. Fatigue crack propagation and overload damaging micromechanisms in a ferritic-pearlitic ductile cast iron. Fatigue \& Fracture of Engineering Materials \& Structures, 2016, 39 (8): 999-1011.

[10] Fernandino D O, Boeri R. Fracture of pearlitic ductile cast iron under different loading conditions. Fatigue \& Fracture of Engineering Materials \& Structures, 2014, 38(1): 80-90. 
[11] Liu J H, Li G L, Liu G S, et al. Study on the characteristics of damage mechanics of ferrite ductile cast iron under tensile loading. Journal of Hebei University of Technology, 2001, 30 (4): 48-49. (In Chinese).

[12] Bartosiewicz L, Singh I, Alberts F A, et al. The influence of chromium on mechanical properties of austempered ductile cast iron. Journal of Materials Engineering \& Performance, 1995, 4(1): 90-101.

[13] Han S Y, Sohn S S, Sang Y S, et al. In Situ fracture observation and fracture toughness analysis of pearlitic graphite cast irons with different nodularity. Metals \& Materials International, 2013, 19(4): 673-682.

[14] Guo Er-jun, Song L, Wang L P, et al. Effects of forced cooling on mechanical properties and fracture behavior of heavy section ductile iron. China Foundry, 2015,12 (6): 398-405.

[15] Song L, Guo Erjun, Tan C L. Effect of Bi on graphite morphology and mechanical properties of heavy section ductile cast iron. China Foundry, 2014,11 (2): 125-131.

[16] Bian G X, Chen Y L, Zhang Y, ea al. Study on stress concentration coefficient of high strength aluminum alloy microstructure. Materials Science \& Technology. 2017, 25 (5): 41-47. (In Chinese).
[17] Yang Z L, He B P, Yang Q Y. Dynamic analysis on a circular inclusion in a radially inhomogeneous medium. Chinese Journal of Theoretical and Applied Mechanics, 2015, 47 (3): 539-543. (In Chinese).

[18] Walter D, Pilkey, Deborah F. Pilkey. Peterson's stress concentration factors. 3rd, John Wiley \& Sons, Inc., Hoboken, New Jersey, 2007: 214.

[19] Yang L H, Zhu H, Tan D L. Influence of soft filler on stress concentration factor of elliptic holes in a rectangular plate. Transactions of Tianjin University. 2012.18: 117-120.

[20] Ping X C, Chen M C. A hybrid-stress FEM for elliptical inclusion problems. Chinese Journal of Solid Mechanics, 2010 31 (1): 60-66 (in Chinese).

[21] Tohru N, Minoru H, Toshio S. Notch effects on impact and bending characteristics of spheroidal graphite and compacted vermicular graphite cast irons with various matrices. Key Engineering Materials, 2011, 457: 392-397. 